\title{
Alleviation of Disease Activity in Lupus mice by Blocking Secondary Pyroptosis of Renal Tubular Epithelial Cells
}

Guihu Luo

Southern Medical University

Fangyuan Yang

Southern Medical University

Zeqing Zhai

Southern Medical University

Yi He

Southern Medical University

Jiaochan Han

Southern Medical University

Lili Zhuang

Southern Medical University

Yanang Zhang

Southern Medical University

Yehao Li

Southern Medical University

Rui Song

Southern Medical University

Xiaoqing Luo

Southern Medical University

Jianheng Liang

Southern Medical University

Erwei Sun ( $\nabla$ sunew@smu.edu.cn )

Southern Medical University https://orcid.org/0000-0001-5664-513X

Research article

Keywords: SLE, GSDME, pyroptosis, JNK, SP600125, caspase-3

Posted Date: January 7th, 2021

DOl: https://doi.org/10.21203/rs.3.rs-140006/v1 
License: (c) (i) This work is licensed under a Creative Commons Attribution 4.0 International License. Read Full License 


\section{Abstract}

Background:Increased apoptosis and/or defect in clearance apoptotic cells resulting in massive secondary necrosis have been recognized as the main causes of systemic lupus erythematosus. Recent findings have revealed that GSDME, a membrane pore protein downstream of caspase-3, is a key mediator of secondary pyroptosis and cellular membrane disruption. Here, we aim to investigate the effects of secondary pyroptosis on disease activity in lupus mice.

Methods:In vivo, Pristane-induced lupus mice were treated with JNK inhibitor SP600125 or vehicle. The disease severity was evaluated and the expression of GSDME and cleaved-caspase-3 detected. In vitro, HK2 human tubular epithelial cells were pretreated with SP600125, followed by treatment with apoptosis inducer, $\mathrm{TNF}+\mathrm{CHX}$, or SLE sera. And then the secondary pyroptosis were examined.

Results:In vivo, high levels of GSDME expression were revealed in renal tubules in PIL mice and SLE patients. In lupus mice, SP600125 administration effectively ameliorated lupus-like features and importantly, reduced the expression of GSDME and cleaved caspase-3 in renal tubules. In vitro, treatment with $\mathrm{TNF}+\mathrm{CHX}$ or SLE sera induced HK2 cells to undergo secondary pyroptosis in a caspase-3-GSDME dependent manner. Likewise, SP600125 significantly reduced GSDME expression and decreased the secondary pyroptosis in HK2 cells.

Conclusions:The GSDME-mediated pyroptosis may be one of the pathogenesis of SLE and targeting GSDME may be a potential strategy for treating SLE.

\section{Introduction}

Systemic lupus erythematosus (SLE) is a complex autoimmune disease involving multiple organs[1].The overall incidence of SLE ranges from 30.0 to 37.6 per 100,000 per year in China[2].To date, extensive studies have been conducted on the pathogenesis and treatment of SLE, but the molecular mechanism of SLE pathogenesis remains unclear. Increased apoptosis and defective clearance are observed in SLE and these apoptotic cells are more prone to progress to secondary necrosis. Many previous studies have demonstrated that secondary necrosis of apoptotic cells plays an important role in the pathogenesis and development of SLE[3-5].

Cell death plays an important role in the maintenance of homeostasis and immune response, and different ways of cell death have different effects on immune response. More than 10 years ago, our research group proposed the "Cell death" recognition model[6, 7]that emphasized the different patterns of cell death in determining the outcomes of immune responses. Necrotic cells initiate the secretion of proinflammatory cytokines to activate immune response, while apoptotic cells induce immune tolerance by inducing anti-inflammatory cytokines. Although apoptotic cells themselves induce immune tolerance, secondary necrosis occurs when apoptotic cells are not cleared timely and effectively, which can significantly promote inflammatory factors release, enhance immune response, and participate in the pathogenesis of SLE[5].Therefore, preventing the secondary necrosis of apoptotic cells and maintaining 
the cells in the apoptotic stage may become one of the choices to prevent autoimmune diseases such as SLE.

Secondary necrosis after apoptosis was once considered to be a passive and uncontrollable process of cell death due to the exhaustion of cell energy, failing to maintain osmotic pressure[8, 9]. However, recent findings revealed that secondary necrosis after apoptosis was also a programmed process called pyroptosis that was mediated by GSDME[10,11]. In the GSDME-high cells, a previously presumed key apoptosis inducer, activated caspase-3, cleaves GSDME to generate the GSDME-N domain. Subsequently, the GSDME-N domain assembles in cellular membrane to form pores, thereby resulting in secondary necrosis. This finding is very encouraging because it opens a window to prevent the secondary necrosis of apoptotic cells and raises new hope for the treatment of SLE.

C-jun N-terminal kinases (JNKs) are a member of MAPK kinases,and they can be activated by different stress stimuli and have various regulatory functions[12].

JNKs are involved in the development and progression of inflammatory diseases[13].

Importantly, studies have shown that increased JNK activation associated with disease activity and organ injury in patients with SLE[14]. Interestingly, one study has found that SP600125,a JNK inhibitor, can prevent GSDME-mediated cell death[15], suggesting that JNK may be a target for regulating GSDME.

Therefore, this study mainly aimed to investigate whether inhibiting JNK could reduce secondary pyroptosis and thus alleviated the disease condition of pristane-induced lupus mice.

\section{Materials And Methods}

\section{Materials}

Rabbit polyclonal antibodies recognizing cleaved Caspase 3 and the N-terminal of GSDME were purchased from Abcam(Catalogue No.ab13847 and No.ab175614,Cambridge, UK). Rabbit monoclonal antibodies to GSDME-N-terminal or JNK1+JNK2+JNK3 or JNK1 + JNK2 + JNK3 (phospho T183+T183+T221) also from Abcam(Catalogue No.ab215191 or No.ab179461 or No.ab124956,Cambridge, UK).TNF-a was purchased from InvivoGen (Catalogue No.rcyc-htnfa,San Diego, USA).Cycloheximide(CHX) was purchased from Sigma-Aldrich (Catalogue No.66-81-9,St.Louis, MO, USA).TNF- $a+$ CHX acts as an inducer of apoptosis[11].The JNK inhibitor SP600125 was purchased from Selleck(Catalogue No.S1460, Houston, TX, USA).

\section{Cell culture}

Human tubular epithelial cell line (HK2) was from Bio-Rad Laboratories (Shanghai, China) and the cells cultured in DNME/F12 media containing 10\% FBS and 1\% penicillin streptomycin. The cells were stimulated with TNF-a $(20 \mathrm{ng} / \mathrm{ml})$ and $\mathrm{CHX}(10 \mu \mathrm{g} / \mathrm{ml})$ to induce cell death, with or without pretreatment

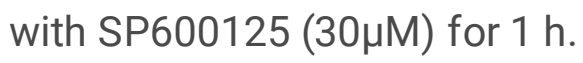




\section{LDH release assay}

After TNF- $a$ and $\mathrm{CHX}$ treatment, the activity of LDH released into cell culture supernatants was measured by using the LDH release kit (Promega) according to the manufacturer's protocol. The supernatant LDH activity was expressed as a percentage of total LDH in the cell lysate.

\section{Microscopy}

To examine the morphological changes of apoptotic or secondary pyroptotic cells, HK2 cells were seeded in the 6-well plates and subjected to indicated treatments. Bright field cell images were captured using the Optical inverted microscope (Olympus, Japan).All the image data displayed represents at least three random field of view.

\section{HK2 cell Hoechst/PI fluorescent staining}

HK2 cells were seeded in the 24-well plates and treated with TNF-a and CHX. Then, cells were stained with Hoechst (Beyotime, China) and PI(BD, USA).Images were collected with a fluorescence microscope(Carl Zeiss, Germany).

\section{Immunofluorescent analysis of GSDME}

After treatment with TNF- $a$ and CHX, the expression of GSDME was detected by immunofluorescence. Cells were fixed with $4 \%$ paraformaldehyde, permeabilized with $0.2 \%$ Triton X-100, and blocked with $5 \%$ fetal bovine serum (FBS). Then, the cells were stained with rabbit anti-GSDME antibody, followed by incubation with a Cy3-conjugated goat secondary antibody against rabbit IgG (Servicebio,China,).Finally, the cells were stained with DAPI(Thermo Fisher

Scientific, USA). Images were captured and analyzed with a fluorescence microscope (Carl Zeiss, Germany).

\section{Western blot}

Cells or tissues were lysed in RIPA protein lysate (Beyotime, China). Cell lysates were fractionated by SDSpolyacrylamide gel electrophoresis (SDS-PAGE) and then transferred to PVDF membranes (Roche, Swit). Blots were probed with appropriate antibodies. Expressions of full length-GSDME and GSDME-N-terminal were detected using anti-GSDME antibodies. Data were analyzed by Image J Software.

\section{Pristane induced lupus (PIL) models}

As described previously,female BALB/ C mice at 6-8 weeks were intraperitoneally injected with $0.5 \mathrm{ml}$ Pristane(Sigma-Aldrich)[16]. Urine protein was detected at 6 weeks after modeling, and urine protein score measured once every two weeks. Albustix test paper was used to determine the urinary protein. The mice were stimulated to urinate by gentle massage of the abdomen, and urine was collected. The fresh urine was dropped into the reaction area of the test paper, and the results were read within $1 \mathrm{~min}$. The urine 
protein score was determined according to the comparison between the color degrees of the reaction area and the standard color band. If urine protein score was more than 1 point in two consecutive tests, it was decided that the modeling was successful.

PIL mice were divided into three groups, named vehicle only control group, PIL group ( $n=9)$, and PIL+SP600125 group(n=9) respectively. Six normal mice were treated with vehicle as control group. In the PIL group, mice received the same amount of vehicle solution (DMSO and PBS). In PIL+SP600125 group, mice were injected intraperitoneally with SP600125 dissolved in $2 \%$ dimethyl sulfoxide (DMSO) in PBS at a dose of $30 \mathrm{mg} / \mathrm{kg}$ body weight once per day [17].

\section{Immunofluorescence analysis of GSDME/Caspase-3 p17}

The frozen kidney sections were blocked with $5 \%$ fetal bovine serum and then stained with rabbit antiGSDME antibody(Abcam, Catalogue No.ab215191) and rabbit anti-caspase3 p17 antibody (Abcam, Catalogue No.ab13847), followed by staining with a second Cy3-conjugated goat anti-rabbit immunoglobulin G (IgG) antibody (Servicebio, Catalogue No.GB21303).For assessment of mouse lgG deposition in kidneys, the frozen kidney sections were stained with Alexa Fluor 555-conjugated goat antimouse IgG(Abcam, Catalogue No.b150114).

\section{Assessment of histopathological changes}

Renal tissues were fixed with $10 \%$ formalin and paraffin-embedded for tissue sectioning. The sections were then stained with hematoxylin and eosin (H\&E). Histopathological changes were examined by pathologists unaware of the experimental information. Austin score to grade lupus disease activity was used as described previously [18].

\section{Statistical analysis}

All data were expressed as mean \pm standard deviation, analyzed by SPSS 20.0 software, and plotted by GraphPad Prism 7.0. One-way ANOVA was used for the comparison of the mean between groups. The difference was considered statistically significant where $p<0.05$ between comparisons.

\section{Results}

\section{Increased GSDME expression in the kidneys of SLE patients and PILmice}

Lupus nephritis, as the main clinical manifestation of SLE[19],develops in most SLE patients within 5 years of diagnosis[20].Therefore, we tested the kidney specimens of SLE patients and PIL mice.

In the kidneys of SLE patients or PIL mice, the renal tubulointerstitium was infiltrated by a large number of inflammatory cells, with disrupted renal tubules, glomerular atrophy and interstitial fibrosis(Fig.1A and C). Importantly, GSDME protein was highly expressed in renal tubules (Fig.1B) in SLE patients and PIL mice (Fig.1D-E). 


\section{TNF-a and CHX induced secondary pyroptosis in HK2 cells}

It has been known that GSDME plays a key role in the secondary pyroptosis[10, 11]. Given that high levels of GSDME were found in renal tubules of lupus kidneys, next we investigated whether renal tubules epithelial cells could develop secondary pyroptosis. In vitro, human tubular epithelial cells (HK2 cells) were treated with the previous presumed apoptosis inducer (TNF-a+CHX)[11], and the secondary pyroptosis of HK2 cells examined.

After the treatment of TNF- $\mathrm{a}+\mathrm{CHX}$, necrotic HK2 cells were significantly increased(Fig.2 A and C), and the dying cells showed evident swelling with characteristic large bubbles protruding from the plasma membrane(Fig.2 B).What's more, TNF-a+CHX treated HK2 cells showed elevated expression of activated caspase-3 and GSDME-N (Fig.2 D). These results confirmed that TNF-a and CHX induced HK2 cells to undergo secondary pyroptosis. When siRNA was used to silence GSDME expression(Fig.2 E),secondary pyroptosis was significantly reduced(Fig.2 F and G).

\section{SP600125 inhibited secondary proptosis}

JNK is a member of MAPK kinases, and it is involved in the pathological process of inflammation. Previous study has reported that the JNK inhibitor SP600125 blocks GSDME-mediated cell death [21]. Next, we investigated the effect of SP600125 on secondary pyroptosis of HK2 cells. We found that SP600125 significantly decreased the secondary pyroptosis, manifested by decreased LDH release (Fig.3 A), diminished cell swelling (Fig.3 B) and reduced numbers of PI positive cells(Fig.3 C). Interestingly, GSDME expression increased after TNF-a+CHX treatment, but decreased significantly when SP600125 was applied (Fig.3 D). It has been known that the total GSDME includes full length GSDME(GSDME-FL) and GSDME-N-terminal(GSDME-N). We found that SP600125 inhibited the production of total GSDME and GSDME-N(Fig.3 E) , suggesting that SP600125 can inhibit both expression and activity of GSDME. These results confirmed that SP600125 significantly blocked the secondary pyroptosis of HK2 cells by reducing the GSDME mediated cell signaling.

\section{Sera of lupus patients increased GSDME expression}

As we found that GSDME expression increased in renal tubular epithelial cells of lupus patients, it is important to know the mechanisms underlying the increased GSDME expression. To this end, HK2 cells were stimulated with serum from lupus patients and examined GSDME expression. The sera of lupus patients or healthy controls were prepared, and then added them to the medium to prepare $10 \%$ cell culture medium. HK2 cells were cultured in prepared medium for $72 \mathrm{~h}$. Interestingly, we found the serum of lupus patients significantly enhanced the expression of GSDME(Fig.4 A and B) and secondary pyroptosis(Fig.4 C). Likewise, SP600125 treatment reduced serum-induced GSDME expression and secondary pyroptosis of HK2 cells(Fig.4 C).

\section{SP600125 effectively attenuated disease activity in PIL mice}


Mice intraperitoneally administered pristane develop autoantibodies and clinical manifestations similar to those of SLE[22, 23]. One study demonstrated that pristane-induced increased apoptosis, provided autoantigens and initiated immune response that led to the development of lupus like autoimmunity[24]. To know whether SP600125 could attenuate SLE activity, we treated PIL mice with SP600125 for 16 weeks. Importantly, SP600125 treatment resulted in not only reduced proteinuria (Fig. 5A), but also decreased levels of anti-dsDNA and anti-Sm antibodies (Fig. 5B). In addition, kidney H\&E staining revealed that SP600125 treatment alleviated renal injury (Fig. 5C), decreased the deposition of IgG (Fig. 5D), and declined plasma IL-6 and TNF-a levels (Fig. 5E).Taken together, these findings suggested that SP600125 treatment could alleviate lupus-like features in PIL mice.

\section{SP600125 inhibited GSDME-mediated secondary pyroptosis in PIL mice}

Compared with the control group , the expression of GSDME in renal tubular epithelial cells in the PIL mice group was significantly increased(Fig.6A), as well as the expression of cleaved caspase3(Fig.6B).Importantly, lupus-prone mice treated with SP600125 showed a reduction in the protein expression levels of GSMDE and cleaved caspase-3(Fig.6A and B). Particularly, abundant GSDME-N in kidney were easily detected in vehicle-treated PIL mice, but rarely found in SP600125-treated PIL mice (Fig. 6C). Based on these results, SP600125 inhibited GSDME-mediated secondary pyroptosis in kidney.

\section{Discussion}

Systemic lupus erythematosus (SLE) is an autoimmune disease characterized by the presence of nuclear autoantibodies,and involved in multiple organs[25]. Although the survival rate of SLE patients has improved in the past decade [26], recent advances have not reduced mortality or the development of endstage lupus nephritis $[27,28]$, which suggests that further study on the pathogenesis of SLE is of great clinical value.

In this study, high levels of GSDME were revealed in renal tubules from SLE patients. Meanwhile, the expressions of GSDME and cleaved caspase-3 in renal tubules of Pristane-induced lupus (PIL) mice were also increased, suggesting that GSDME-mediated secondary pyroptosis may play an important role in the pathogenesis and development of SLE (Figs. 1 and 6).

Subsequently, renal tubular epithelial cells (HK2) were selected as the study objects to verify the mechanism of GSDME-mediated secondary pyroptosis. HK2 cells stimulated with TNF + CHX showed elevated protein expression levels of cleaved caspase-3 and GSDME-N domain and increased secondary pyroptosis(Fig. 2). Importantly, sera of SLE patients also induced HK2 cells to undergo secondary pyroptosis (Fig. 4).

Interestingly, a JNK inhibitor, SP600125, significantly reduced the expression of GSDME and the secondary pyroptosis of HK2 cells (Fig. 3). In vivo, SP600125 administration effectively ameliorated lupus-like features in PIL mice, showed by a reduction in levels of autoantibodies, a decrease in proteinuria and an improvement in pathology of kidneys (Fig. 5). Importantly, PIL mice treated with 
SP600125 showed a reduction in the expression levels of GSMDE and cleaved caspase-3(Fig. 6). Based on these results, we believe that SP600125 effectively attenuates disease activity in PIL mice by inhibiting GSDME-mediated secondary pyroptosis.

GSDME, also known as DFNA5 (Deafness, Autosomal Dominant 5), was first associated with sensorineural hearing loss in humans [29]. Most deafness-causing mutations in GSDME lead to deletion of GSDME C-terminal transcription and could induce spontaneous pyroptosis[30].In addition to hearing loss, GSDME has been linked to many cancers, such as breast[31, 32], epatocellular[33], gastric[34] colorectal[35]cancers. In these cancers, GSDME methylation was significantly increased, resulting in GSDME epigenetic silencing, which reduced GSDME levels[36].GSDME can be regulated by p53 because the presence of p53 binding sites in GSDME intron 1[36]. Treatment with the demethylating agent, such as 5-aza-2'-deoxycytidine, can restore p53-induced GSDME expression[37]. Based on these reports, GSDME is considered to be a tumor suppressor gene because inactivation of GSDME inhibits its necrotic function, thus promoting tumor formation.

GSDME actually has roles in the switch of the cell death mode from apoptosis to secondary pyroptosis[38].Caspase-3 specifically clevages and activates GSDME, resulting in pyroptosis. This changes the concept of programmed cell death as caspase- 3 is long considered a marker of apoptosis.In fact, it is the expression level of GSDME that determines the cell death mode of Caspase-3 activated cells[11].Cells with high GSDME expression undergo pyroptosis upon the stimulation of apoptotic inducer like chemotherapy drug. Whereas, cells lacking sufficient GSDME develop apoptosis.

GSDME may play a role in chemotherapy-induced caspase-3-dependent cell death due to its ability to regulate apoptosis and secondary pyroptosis[11]. In vivo study, Gsdme $\mathrm{G}^{-/-}$mice were protected from chemotherapy drug induced tissue damages and weight loss[11]. Peritoneal injection of cisplatin or 5-FU caused severe small intestinal impairment and infiltration of immune cells in $\mathrm{Gsdme}^{+/+}$mice, whereas these signs of tissue damage were reduced in $\mathrm{Gsdme}^{-/-}$mice[38].Moreover, Gsdme ${ }^{-/-}$mice showed attenuated lung injury and inflammation in response to cisplatin or bleomycin[38].These observations confirmed a significant role of GSDME-mediated cell death in promoting inflammation and organ damage. However, the role of GSDME-mediated secondary pyroptosis in lupus has not been reported. Here, we found that high levels of GSDME were found in kidney specimens from SLE patients and lupusprone mice. We also provided evidence that GSDME-mediated secondary pyroptosis may play an important role in the pathogenesis and development of SLE. Therefore, preventing GSDME-mediated secondary is a new strategy and target for the treatment of SLE.

C-jun N-terminal kinases (JNKs), as a member of the mitogen-activated protein kinases (MAPK) family, regulate physiological processes such as neuronal function, immune function and embryonic development by influencing gene expression, cytoskeleton protein dynamics and cell death/survival pathways[39]. In response to apoptotic stimulation, JNKs regulate the activity of various pro-apoptotic and anti-apoptotic proteins, thus participating in extrinsic and the intrinsic apoptotic pathways[40].Multiple abnormalities of the JNK pathway are involved in several autoimmune diseases, 
including chronic idiopathic urticaria, inflammatory bowel disease, and systemic lupus erythematosus (SLE)[41]. In SLE patients, increased JNK activities correlate with disease activity[42] and long-term organ damage[43]. SP600125 is a common JNKs highly selective inhibitor. Studies have shown that JNKs inhibitors, including SP600125, can significantly alleviate the symptoms of immune diseases[14].One study has found that the JNK inhibitor SP600125 can block GSDME-mediated cell death[15], but the mechanism is unclear. We found that SP600125 could significantly improve the symptoms of lupus mice by inhibiting secondary pyroptosis in two ways. Firstly, SP600125 can inhibit the activation of caspase3 protein and reduce the production of apoptotic cells. Second, SP600125 can reduce the expression and activation of GSDME, which inhibit the secondary pyroptosis, but initiate apoptotic signaling. From this perspective, SP600125 may be an ideal drug for the treatment of lupus.

A limitation of this study should be noted. SP600125 can only indirectly regulate GSDME expression and activation and, currently, there are no specific GSDME inhibitors. Therefore, our next work will focus on the effect of GSDME knockout on lupus mouse model and the search for GSDME-specific inhibitors.

\section{Conclusions}

In conclusion, our data demonstrated that JNK inhibitor SP600125 effectively attenuated disease activity in PIL mice by inhibiting GSDME-mediated secondary pyroptosis. This implies the important role of GSDME-mediated secondary pyroptosis in SLE pathogenesis and development, and suggests that SP600125 may be a potential drug for treating SLE.

\section{Declarations}

\section{Ethics approval and consent to participate}

This study has been approved by the Ethics Committee of The Third Affiliated Hospital, Southern Medical University and all participants signed informed consent forms.

\section{Consent for publication}

Not applicable.

\section{Availability of data and material}

The datasets generated during and/or analyzed during the current study are available from the corresponding author on reasonable request.

\section{Competing interests}

The authors declare that they have no competing interests.

\section{Funding}


This work was supported by grants from National Natural Science Foundation

of China (Grant Nos. 81873880) .

\section{Authors' contributions}

GHL carried out most of the experiments, participated in the analysis of data, and drafted the manuscript. YH participated in the design of the study, data analysis and interpretation. FYY and ZQZ participated in the animal experiments and performed the statistical analysis. $\mathrm{JCH}$ carried out the flow cytometry analysis. LLZ,YNZ,YHL,RS,XQL,JHL participated in the animal experiments. EWS conceived the idea for the project, participated in its design and coordination, and modified the manuscript. All authors read and approved the final manuscript.

\section{Acknowledgements}

Not applicable.

\section{References}

1. Kaul A, Gordon C, Crow MK, Touma Z, Urowitz MB, van Vollenhoven R, Ruiz-Irastorza G, Hughes G. Systemic lupus erythematosus. Nat Rev Dis Primers. 2016;2:16039.

2. Carter EE, Barr SG, Clarke AE. The global burden of SLE: prevalence, health disparities and socioeconomic impact. Nat Rev Rheumatol. 2016;12:605-20.

3. Munoz LE, Lauber K, Schiller M, Manfredi AA, Herrmann M. The role of defective clearance of apoptotic cells in systemic autoimmunity. Nat Rev Rheumatol. 2010;6:280-9.

4. Mahajan A, Herrmann M, Munoz LE. Clearance Deficiency and Cell Death Pathways: A Model for the Pathogenesis of SLE. Front Immunol. 2016;7:35.

5. Mistry P, Kaplan MJ. Cell death in the pathogenesis of systemic lupus erythematosus and lupus nephritis. Clin Immunol. 2017;185:59-73.

6. Sun E. Cell death recognition model for the immune system. Med Hypotheses. 2008;70:585-96.

7. Sun EW, Shi YF. Apoptosis: the quiet death silences the immune system. Pharmacol Ther. 2001;92:135-45.

8. Gleiss B, Gogvadze V, Orrenius S, Fadeel B. Fas-triggered phosphatidylserine exposure is modulated by intracellular ATP. Febs Lett. 2002;519:153-8.

9. Zamaraeva MV, Sabirov RZ, Maeno E, Ando-Akatsuka Y, Bessonova SV, Okada Y. Cells die with increased cytosolic ATP during apoptosis: a bioluminescence study with intracellular luciferase. Cell Death Differ. 2005;12:1390-7.

10. Rogers C, Fernandes-Alnemri T, Mayes L, Alnemri D, Cingolani G, Alnemri ES. Cleavage of DFNA5 by caspase-3 during apoptosis mediates progression to secondary necrotic/pyroptotic cell death. Nat Commun. 2017;8:14128. 
11. Wang Y, Gao W, Shi X, Ding J, Liu W, He H, Wang K, Shao F. Chemotherapy drugs induce pyroptosis through caspase-3 cleavage of a gasdermin. Nature. 2017;547:99-103.

12. Johnson GL, Lapadat R. Mitogen-activated protein kinase pathways mediated by ERK, JNK, and p38 protein kinases. Science. 2002;298:1911-2.

13. Cui J, Zhang M, Zhang YQ, Xu ZH. JNK pathway: diseases and therapeutic potential. Acta Pharmacol Sin. 2007;28:601-8.

14. Guma M, Ronacher LM, Firestein GS, Karin M, Corr M. JNK-1 deficiency limits macrophage-mediated antigen-induced arthritis. Arthritis Rheum. 2011;63:1603-12.

15. Van Rossom S, Op DBK, Hristovska V, Winderickx J, Van Camp G. The deafness gene DFNA5 induces programmed cell death through mitochondria and MAPK-related pathways. Front Cell Neurosci. 2015;9:231.

16. Liao P, He Y, Yang F, Luo G, Zhuang J, Zhai Z, Zhuang L, Lin Z, Zheng J, Sun E. Polydatin effectively attenuates disease activity in lupus-prone mouse models by blocking ROS-mediated NET formation. Arthritis Res Ther. 2018;20:254.

17. Zhou Q, Wang M, Du Y, Zhang W, Bai M, Zhang Z, Li Z, Miao J. Inhibition of c-Jun N-terminal kinase activation reverses Alzheimer disease phenotypes in APPswe/PS1dE9 mice. Ann Neurol. 2015;77:637-54.

18. Austin HR, Muenz LR, Joyce KM, Antonovych TT, Balow JE. Diffuse proliferative lupus nephritis: identification of specific pathologic features affecting renal outcome. Kidney Int. 1984;25:689-95.

19. Pons-Estel GJ, Serrano R, Plasin MA, Espinosa G, Cervera R. Epidemiology and management of refractory lupus nephritis. Autoimmun Rev. 2011;10:655-63.

20. Anders HJ, Saxena R, Zhao MH, Parodis I, Salmon JE, C Mohan: Lupus nephritis. Nat Rev Dis Primers 2020, 6:7.

21. Van Rossom S, Op DBK, Hristovska V, Winderickx J, Van Camp G. The deafness gene DFNA5 induces programmed cell death through mitochondria and MAPK-related pathways. Front Cell Neurosci. 2015;9:231.

22. Satoh M, Kumar A, Kanwar YS, Reeves WH. Anti-nuclear antibody production and immune-complex glomerulonephritis in BALB/c mice treated with pristane. Proc Natl Acad Sci U S A. 1995;92:109348.

23. Satoh $M$, Reeves $W H$. Induction of lupus-associated autoantibodies in BALB/c mice by intraperitoneal injection of pristane. J Exp Med. 1994;180:2341-6.

24. Calvani N, Caricchio R, Tucci M, Sobel ES, Silvestris F, Tartaglia P, Richards HB. Induction of apoptosis by the hydrocarbon oil pristane: implications for pristane-induced lupus. $\mathrm{J}$ Immunol. 2005; 175:4777-82.

25. Durcan L, O'Dwyer T, Petri M. Management strategies and future directions for systemic lupus erythematosus in adults. Lancet. 2019;393:2332-43. 
26. Tsokos GC, Lo MS, Costa RP, Sullivan KE. New insights into the immunopathogenesis of systemic lupus erythematosus. Nat Rev Rheumatol. 2016;12:716-30.

27. Tektonidou MG, Lewandowski LB, Hu J, Dasgupta A, Ward MM. Survival in adults and children with systemic lupus erythematosus: a systematic review and Bayesian meta-analysis of studies from 1950 to 2016. Ann Rheum Dis. 2017;76:2009-16.

28. Tektonidou MG, Dasgupta A, Ward MM. Risk of End-Stage Renal Disease in Patients With Lupus Nephritis, 1971-2015: A Systematic Review and Bayesian Meta-Analysis. Arthritis Rheumatol. 2016;68:1432-41.

29. Laer LV, Huizing EH, Verstreken M, van Zuijlen D, Wauters JG, Bossuyt PJ, Van de Heyning P, McGuirt WT, Smith RJ, Willems PJ, et al. Nonsyndromic hearing impairment is associated with a mutation in DFNA5. Nat Genet. 1998;20:194-7.

30. Gregan J, Van Laer L, Lieto LD, Van Camp G, Kearsey SE. A yeast model for the study of human DFNA5, a gene mutated in nonsyndromic hearing impairment. Biochim Biophys Acta. 2003;1638:179-86.

31. Croes L, Beyens M, Fransen E, Ibrahim J, Vanden BW, Suls A, Peeters M, Pauwels P, Van Camp G, Op DBK. Large-scale analysis of DFNA5 methylation reveals its potential as biomarker for breast cancer. Clin Epigenetics. 2018;10:51.

32. Stoll G, Ma Y, Yang H, Kepp O, Zitvogel L, Kroemer G. Pro-necrotic molecules impact local immunosurveillance in human breast cancer. Oncoimmunology. 2017;6:e1299302.

33. Wang CJ, Tang L, Shen DW, Wang C, Yuan QY, Gao W, Wang YK, Xu RH, Zhang H. The expression and regulation of DFNA5 in human hepatocellular carcinoma DFNA5 in hepatocellular carcinoma. Mol Biol Rep. 2013;40:6525-31.

34. Wang Y, Yin B, Li D, Wang G, Han X, Sun X. GSDME mediates caspase-3-dependent pyroptosis in gastric cancer. Biochem Biophys Res Commun. 2018;495:1418-25.

35. Kim MS, Chang X, Yamashita K, Nagpal JK, Baek JH, Wu G, Trink B, Ratovitski EA, Mori M, Sidransky D. Aberrant promoter methylation and tumor suppressive activity of the DFNA5 gene in colorectal carcinoma. Oncogene. 2008;27:3624-34.

36. Li YQ, Peng JJ, Peng J, Luo XJ. The deafness gene GSDME: its involvement in cell apoptosis, secondary necrosis, and cancers. Naunyn Schmiedebergs Arch Pharmacol. 2019;392:1043-8.

37. Fujikane T, Nishikawa N, Toyota M, Suzuki H, Nojima M, Maruyama R, Ashida M, Ohe-Toyota M, Kai $\mathrm{M}$, Nishidate T, et al. Genomic screening for genes upregulated by demethylation revealed novel targets of epigenetic silencing in breast cancer. Breast Cancer Res Treat. 2010;122:699-710.

38. Yu X, He S. GSDME as an executioner of chemotherapy-induced cell death. Sci China Life Sci. 2017;60:1291-4.

39. Zeke A, Misheva M, Remenyi A, Bogoyevitch MA. JNK Signaling: Regulation and Functions Based on Complex Protein-Protein Partnerships. Microbiol Mol Biol Rev. 2016;80:793-835.

40. Dhanasekaran DN, Reddy EP. JNK signaling in apoptosis. Oncogene. 2008;27:6245-51. 
41. Molad Y, Amit-Vasina M, Bloch O, Yona E, Rapoport MJ. Increased ERK and JNK activities correlate with disease activity in patients with systemic lupus erythematosus. Ann Rheum Dis. 2010;69:17580 .

42. Molad Y, Amit-Vasina M, Bloch O, Yona E, Rapoport MJ. Increased ERK and JNK activities correlate with disease activity in patients with systemic lupus erythematosus. Ann Rheum Dis. 2010;69:17580.

43. Bloch O, Amit-Vazina M, Yona E, Molad Y, Rapoport MJ. Increased ERK and JNK activation and decreased ERK/JNK ratio are associated with long-term organ damage in patients with systemic lupus erythematosus. Rheumatology. 2014;53:1034-42.

\section{Figures}
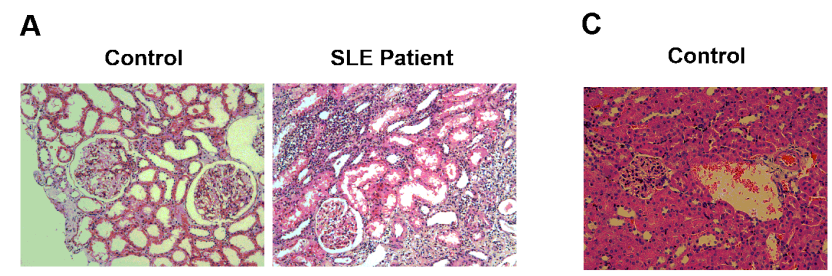

PIL mice

E

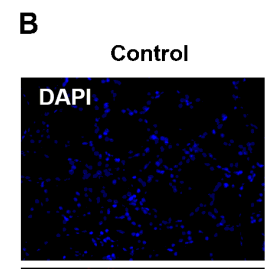

SLE Patient

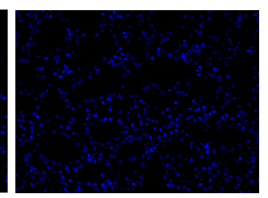

D
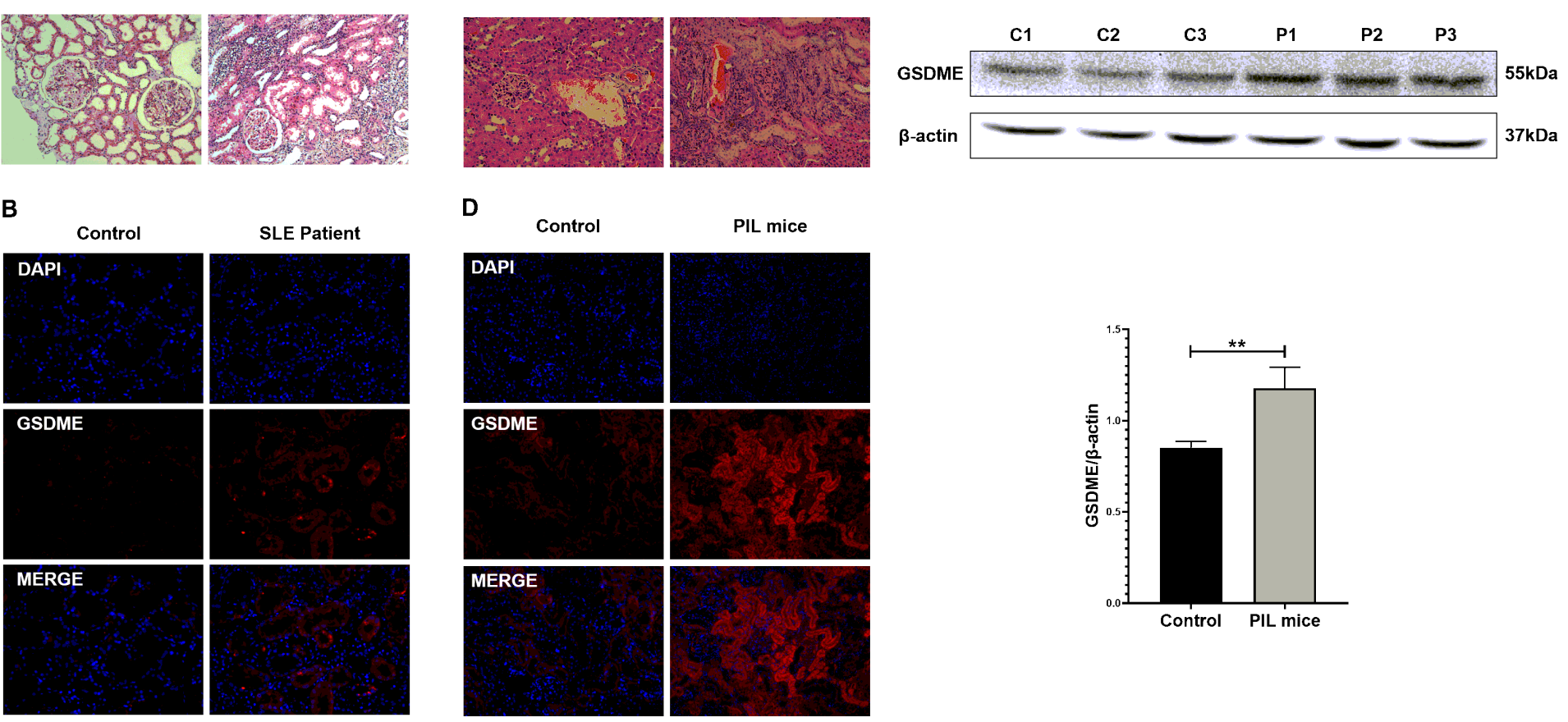

\section{Figure 1}

GSDME was highly expressed in renal tubular cells in SLE patients and Pristane-induced lupus mice. (A) $\mathrm{H}$ \& E staining renal pathological changes of health controls and SLE patients.(B)immunofluorescence analysis of the expressions of GSDME in kidney specimens from health controls and SLE patients. (C)H \& E staining renal pathological changes and (D) immunofluorescence analysis of GSDME expression in control and PIL mice kidney .(E)Western blot (top) and quantitative analysis (bottom) of the expressions of GSDME in control and PIL mice kidney. Data were shown as mean \pm SD, ${ }^{*} \mathrm{p}<0.01$. 


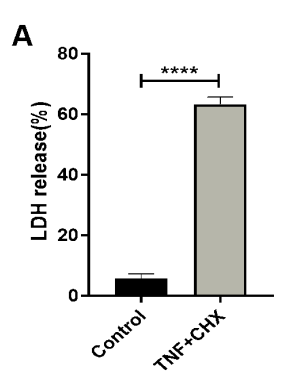

D
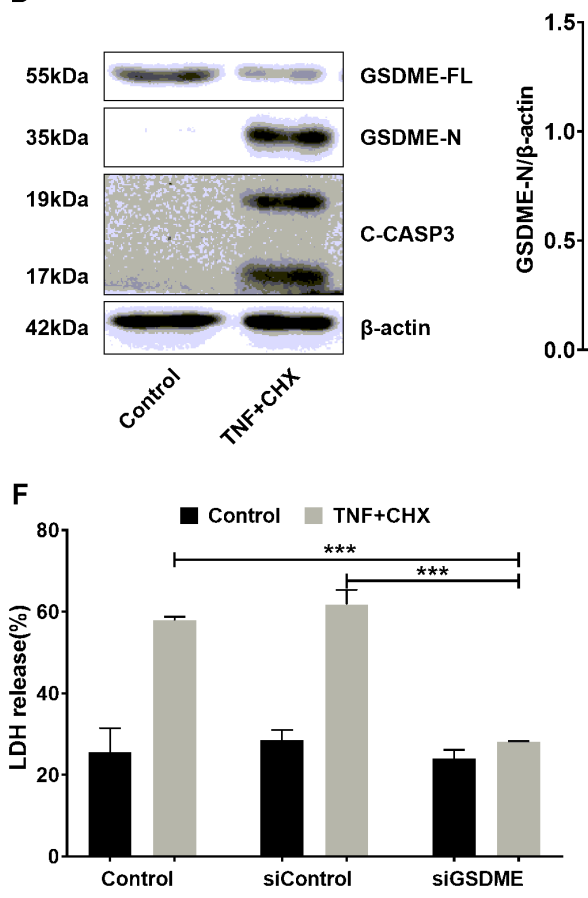

C
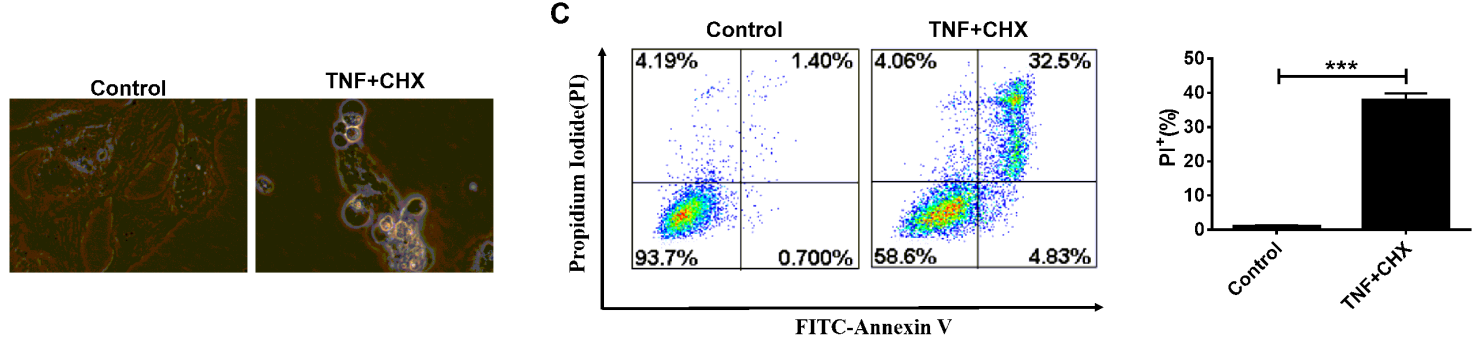

E

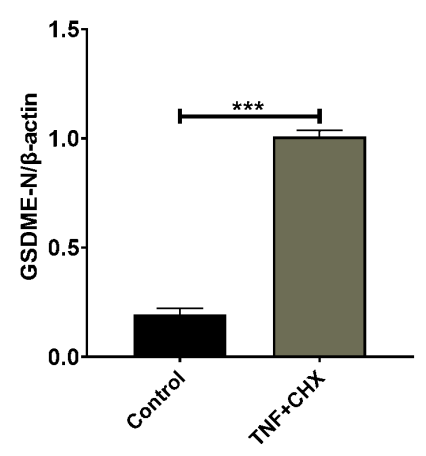

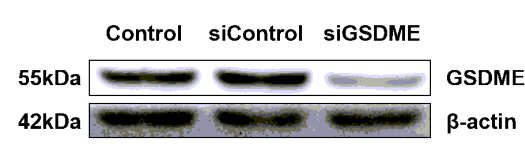

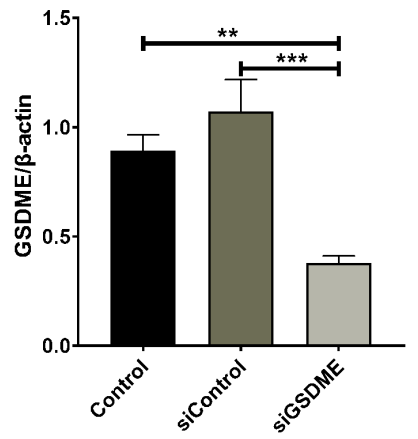

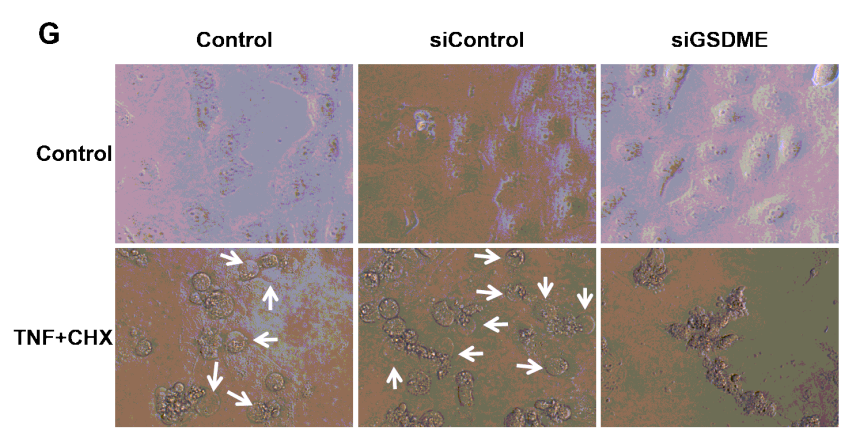

Figure 2

TNF+CHX induced secondary pyroptosis in HK2 cells. (A)HK2 cells were treated with TNF-a and CHX for $12 \mathrm{~h}$, then the cell viability was detected using LDH release.(B) Phase-contrast imaging assay of HK2 cells after TNF-a and $\mathrm{CHX}$ treatment.(C)Flow cytometry of propidium iodide (PI) and Annexin V-FITC-stained cells. (D)Western blot (left) and quantitative analysis (right) of the expressions of GSDME $(n=3)$. (E) HK cells were transfected with NC- or GSDME-siRNA. (F)LDH releases and (G)phase-contrast images of HK2 cells were detected after TNF-a and $\mathrm{CHX}$ treatment. Arrows, the pyroptotic cells. Data were shown as mean $\pm S D,{ }^{* *} p<0.01,{ }^{* \star *} p<0.001,{ }^{* \star * *} p<0.0001$. 
A

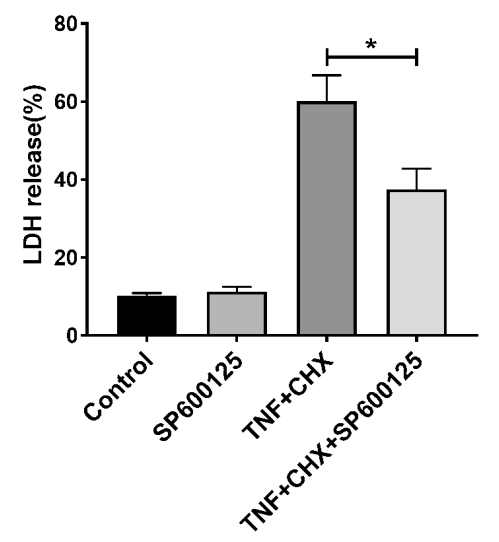

D

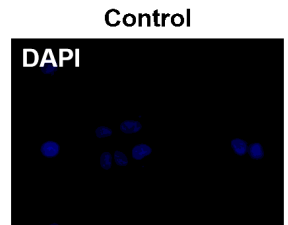

GSDME

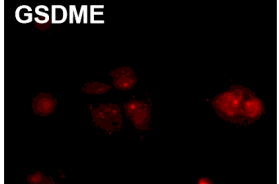

MERGE
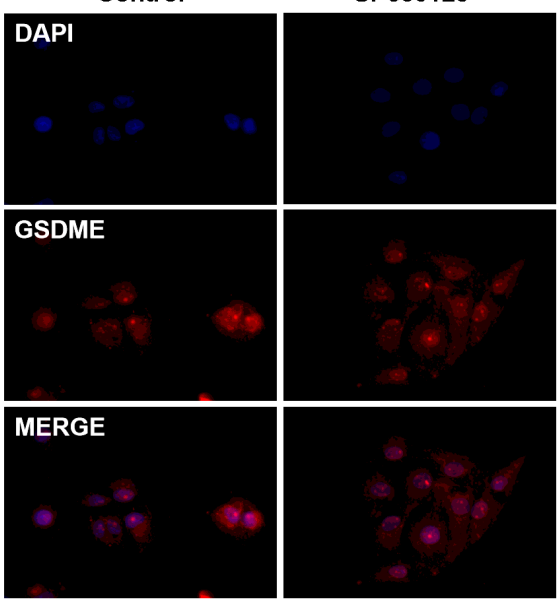

E

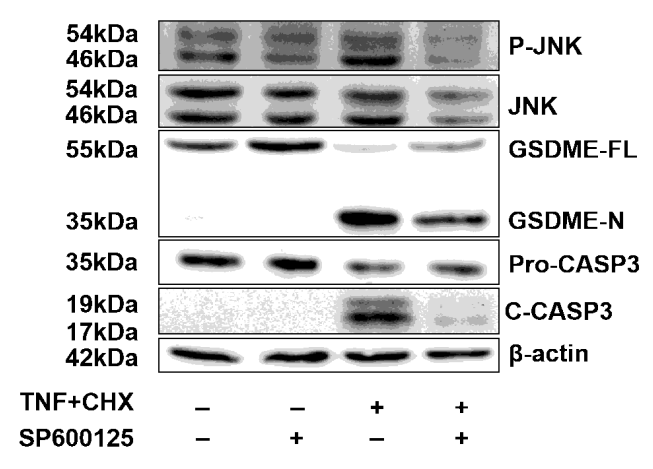

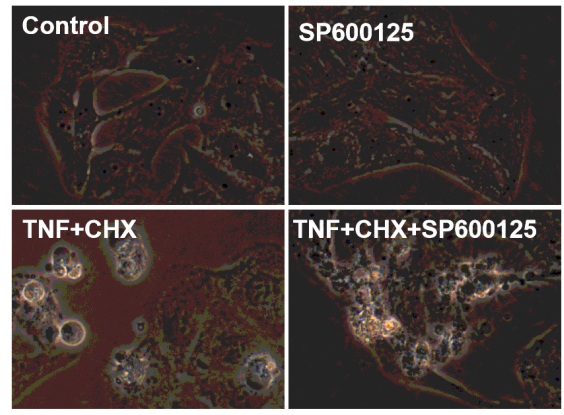

$\mathrm{TNF}+\mathrm{CHX}$

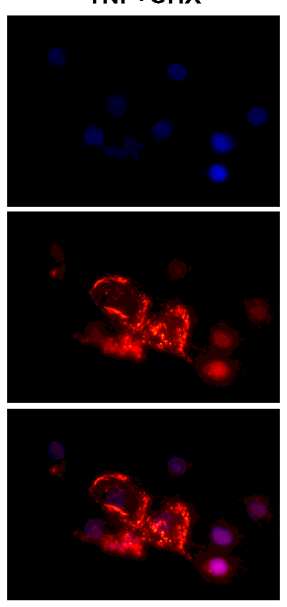

TNF+CHX+SP600125

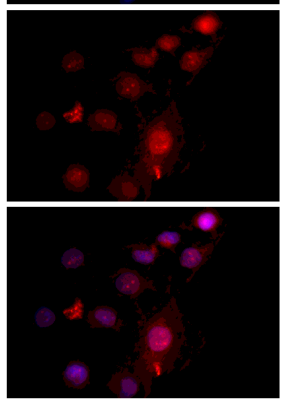

C

Hoechst

PI
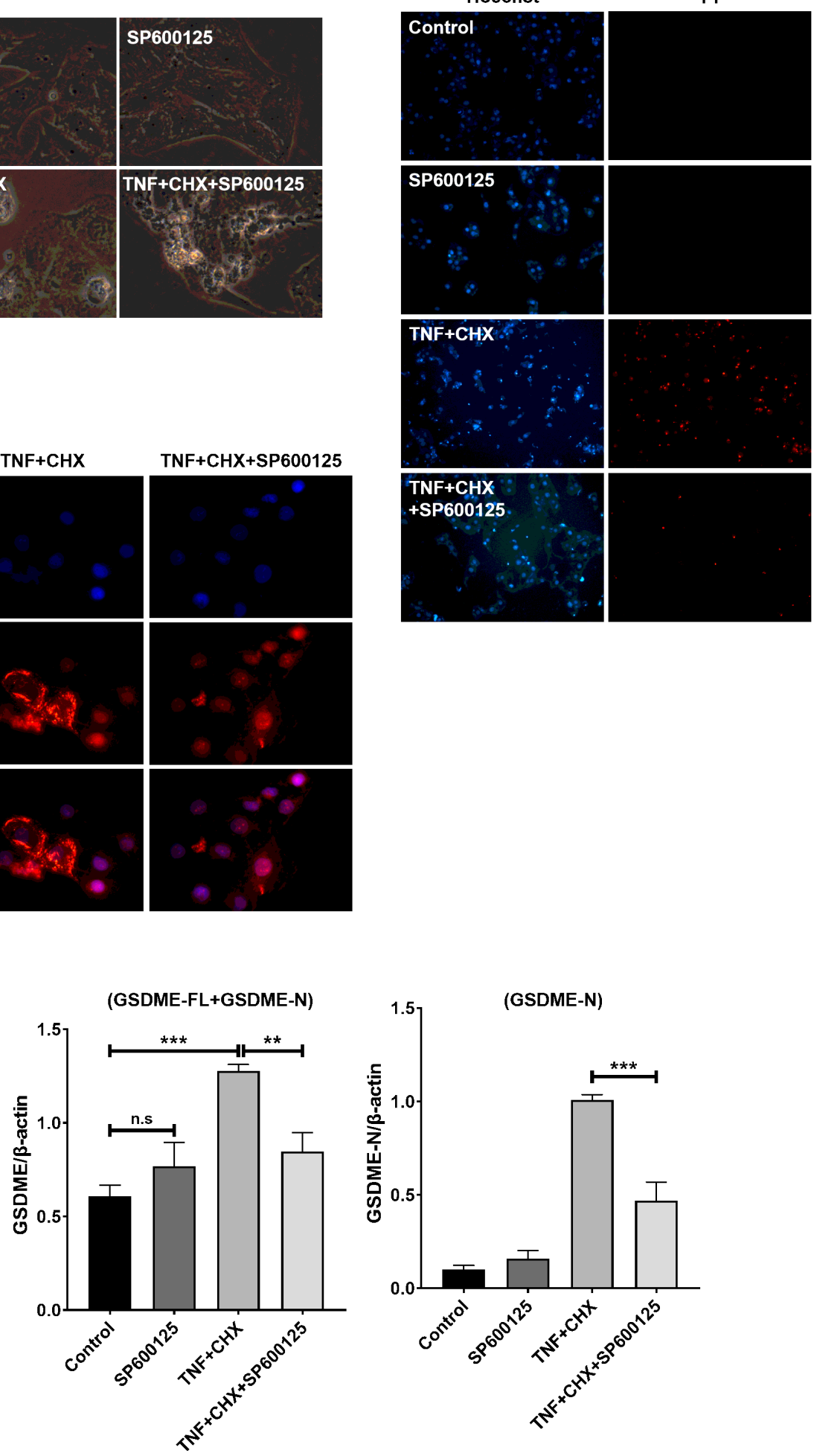

\section{Figure 3}

SP600125 inhibited GSDME mediated secondary pyroptosis in HK cells. HK2 cells were pretreated with SP600125 for $1 \mathrm{~h}$ and then exposed to TNF-a and CHX for 12h. (A)Levels of LDH were detected. (B)Phasecontrast images of HK2 cells were detected. (C)HK2 cells were stained with Hochest(blue) and PI(red).

(D)Immunofluorescence staining for GSDME(red) was assessed.(E)Expression of GSDME-FL and 
GSDME-N were detected by western blot. Data were shown as mean \pm SD, ${ }^{*} p<0.05$, ** $p<0.01$, ${ }^{\star \star *} p<$ 0.001 .

A

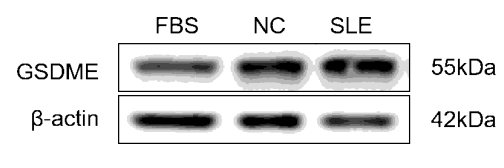

B

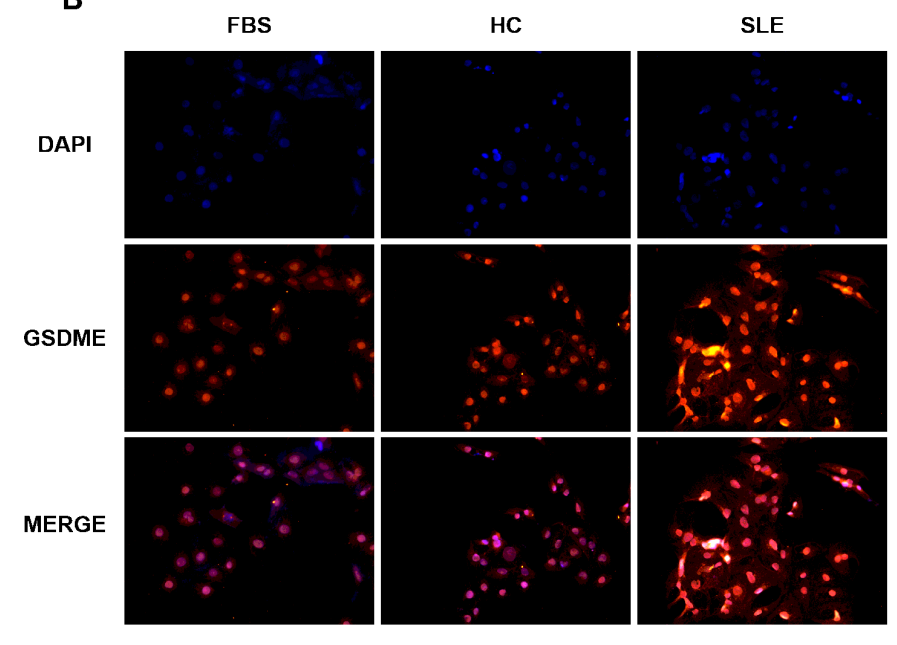

C
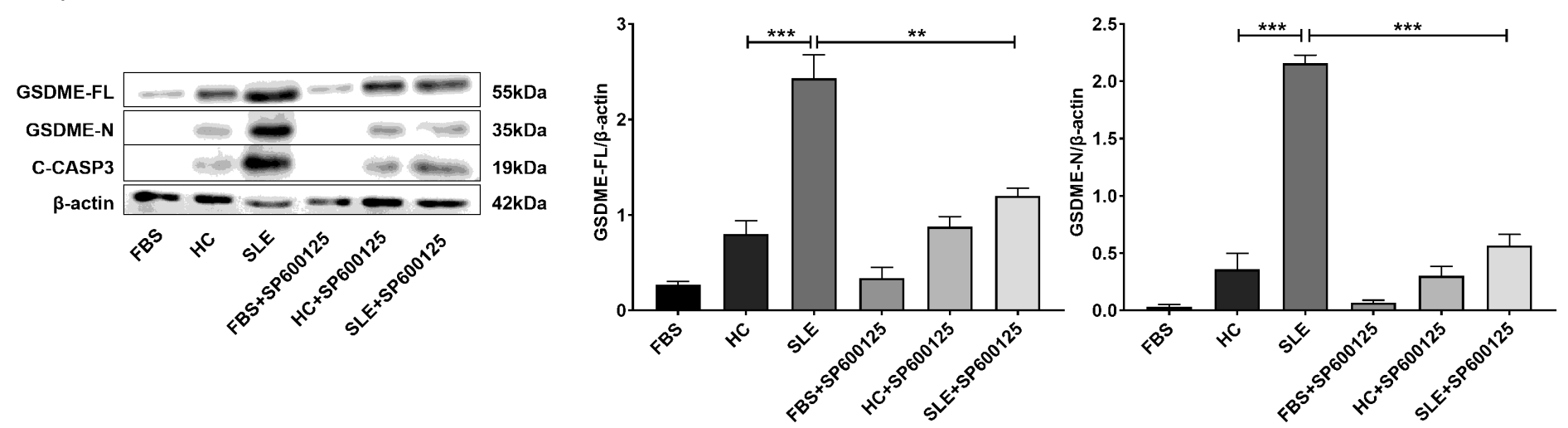

Figure 4

Sera of lupus patients induced high expression of GSDME in HK cells. The sera of lupus patients $(n=5)$ or healthy controls $(n=3)$ were mixed together, and then added them to the medium to prepare $10 \%$ cell culture medium.HK2 cells were cultured in prepared medium for 72h.(A)Western blot (left) and quantitative analysis (right) of the expressions of GSDME.(B)HK2 cells were immunofluorescent stained by DAPI(blue) and GSDME(red).(C)HK2 cells were pretreated with SP600125 for $1 \mathrm{~h}$ and then exposed to the serum of lupus patients or normal controls, western blot and quantitative analysis of the expressions of GSDME. Data were shown as mean \pm SD, ${ }^{\star *} p<0.01$, ${ }^{\star \star *} p<0.001$. 
A

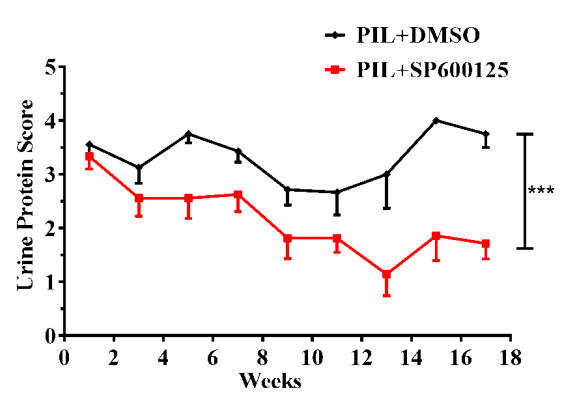

B

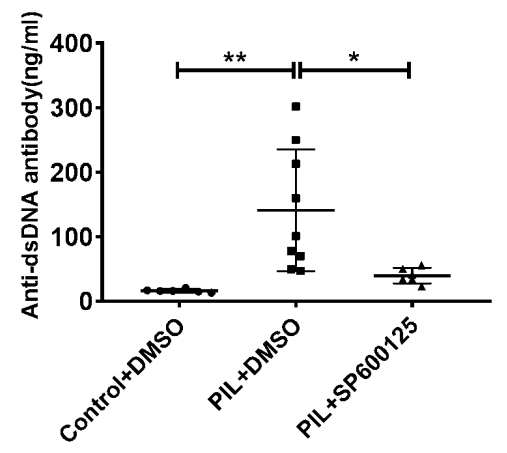

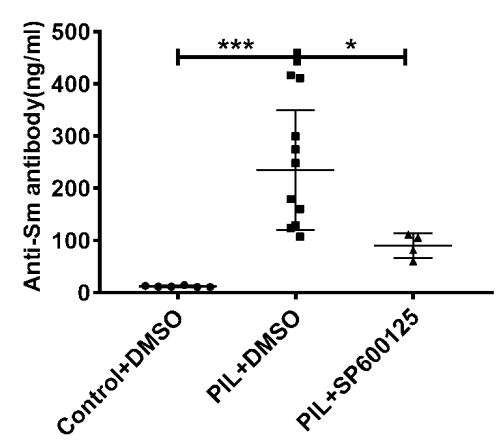

C

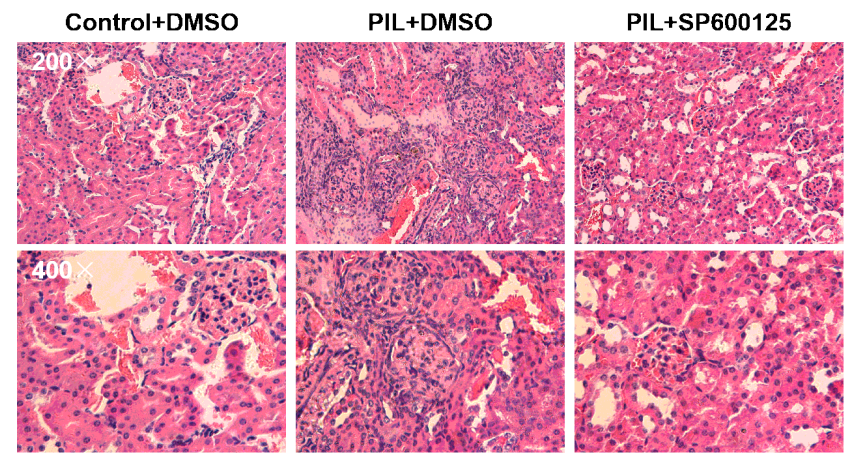

D

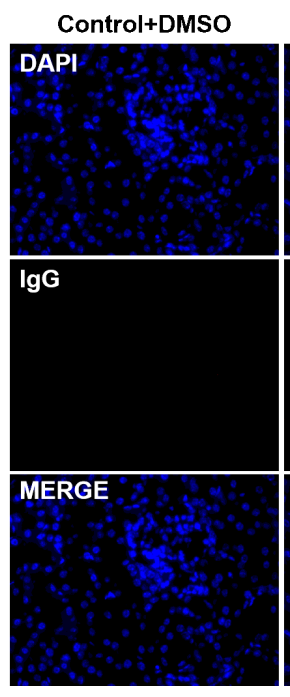

PIL+DMSO

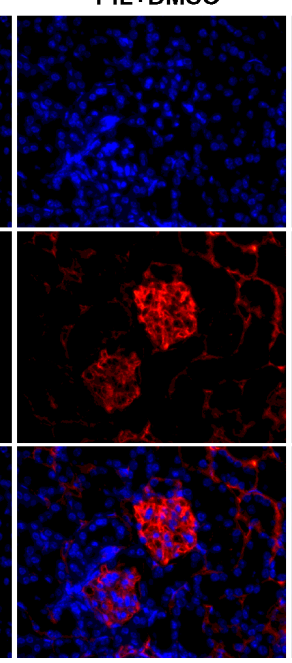

PIL+SP600125

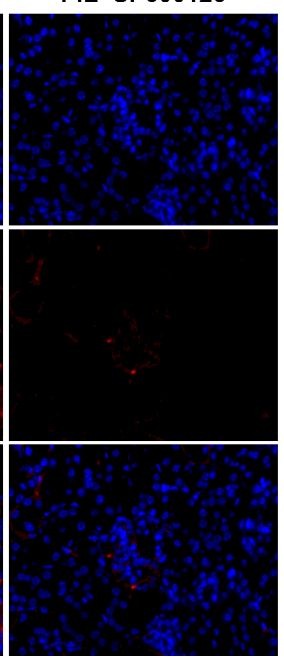

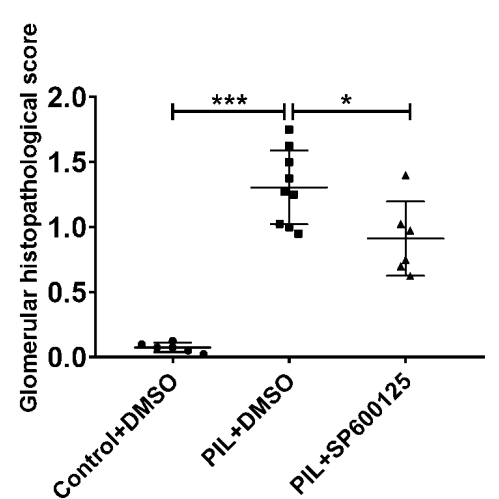

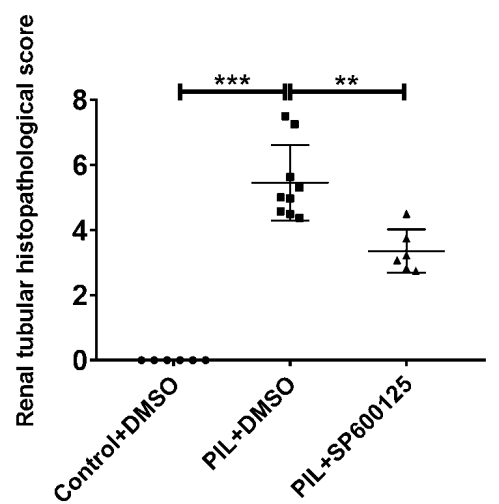

E
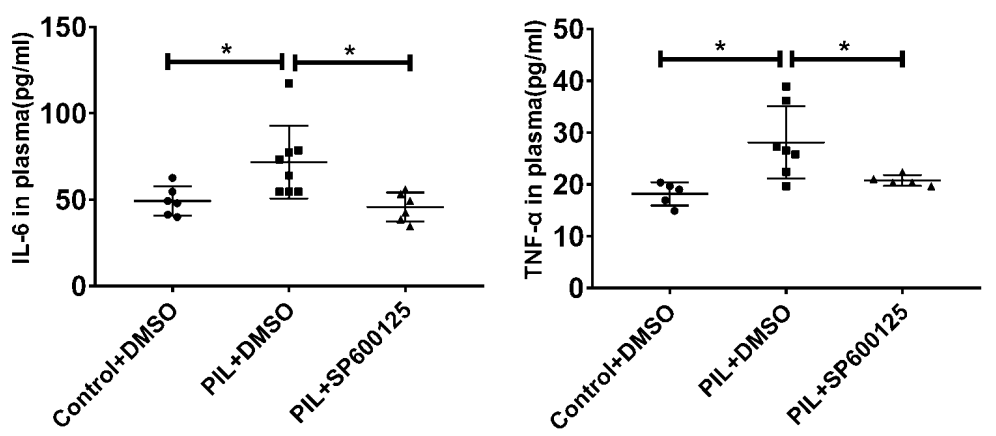

\section{Figure 5}

SP600125 effectively attenuated disease activity in PIL mice. (A)Albustix test paper was used to determine the urinary protein. Urine protein score was measured once every two weeks. (B,E)The levels of autoantibodies(anti-dsDNA antibody and anti-Sm antibody) and cytokines(IL-6 and TNF-a) were examined by ELISA kit.(C)Representative H\&E staining of glomerular and renal tubular lesions in kidneys were shown (left). Austin scores of kidneys were determined (right).(D)Immunofluorescence showed IgG deposition in the kidney. Data were shown as mean $\pm S D,{ }^{*} p<0.05$, ${ }^{\star \star} p<0.01$, ${ }^{\star \star \star} p<0.001$. 
A
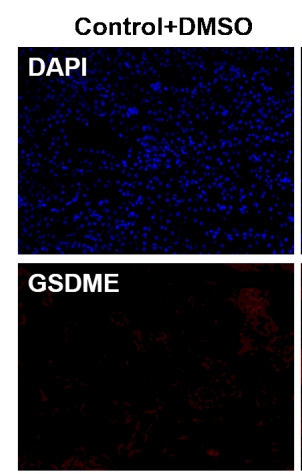

MERGE

C
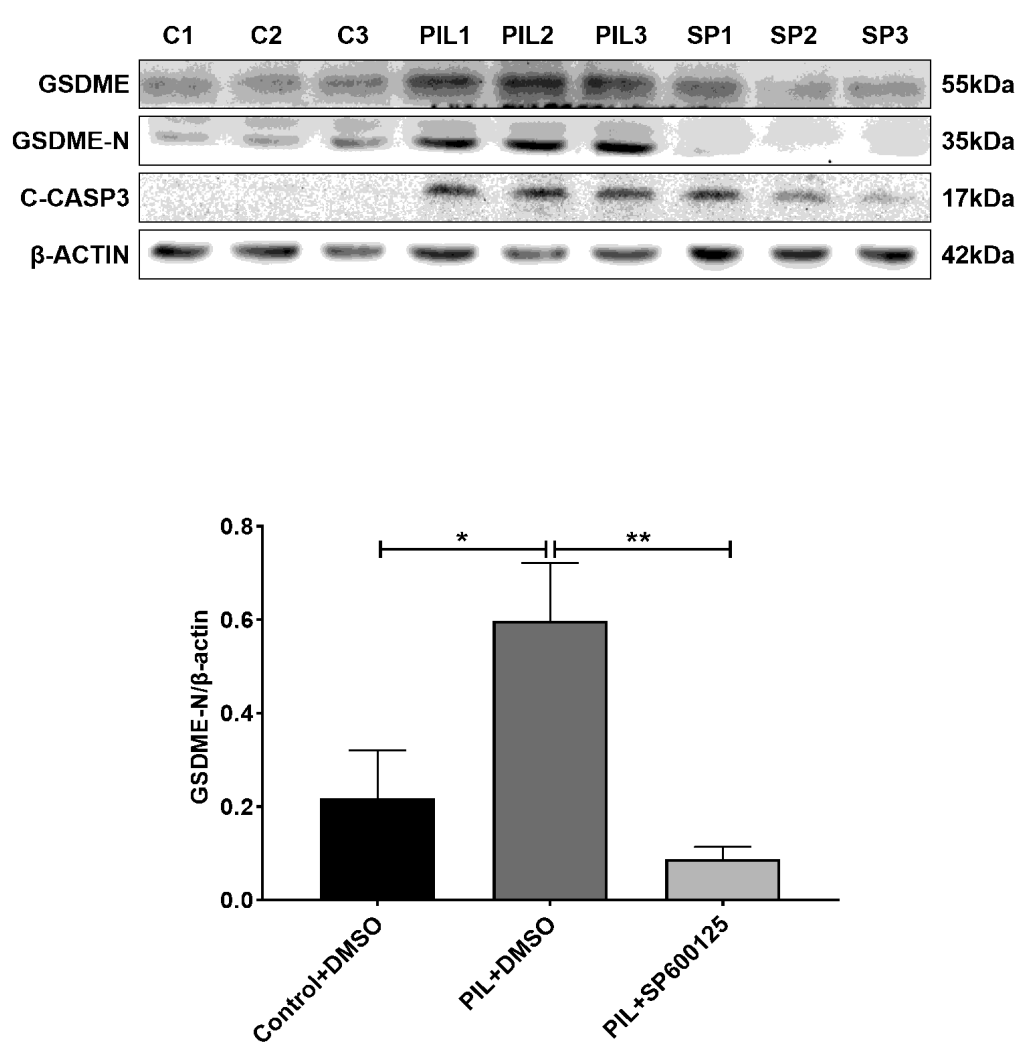

B
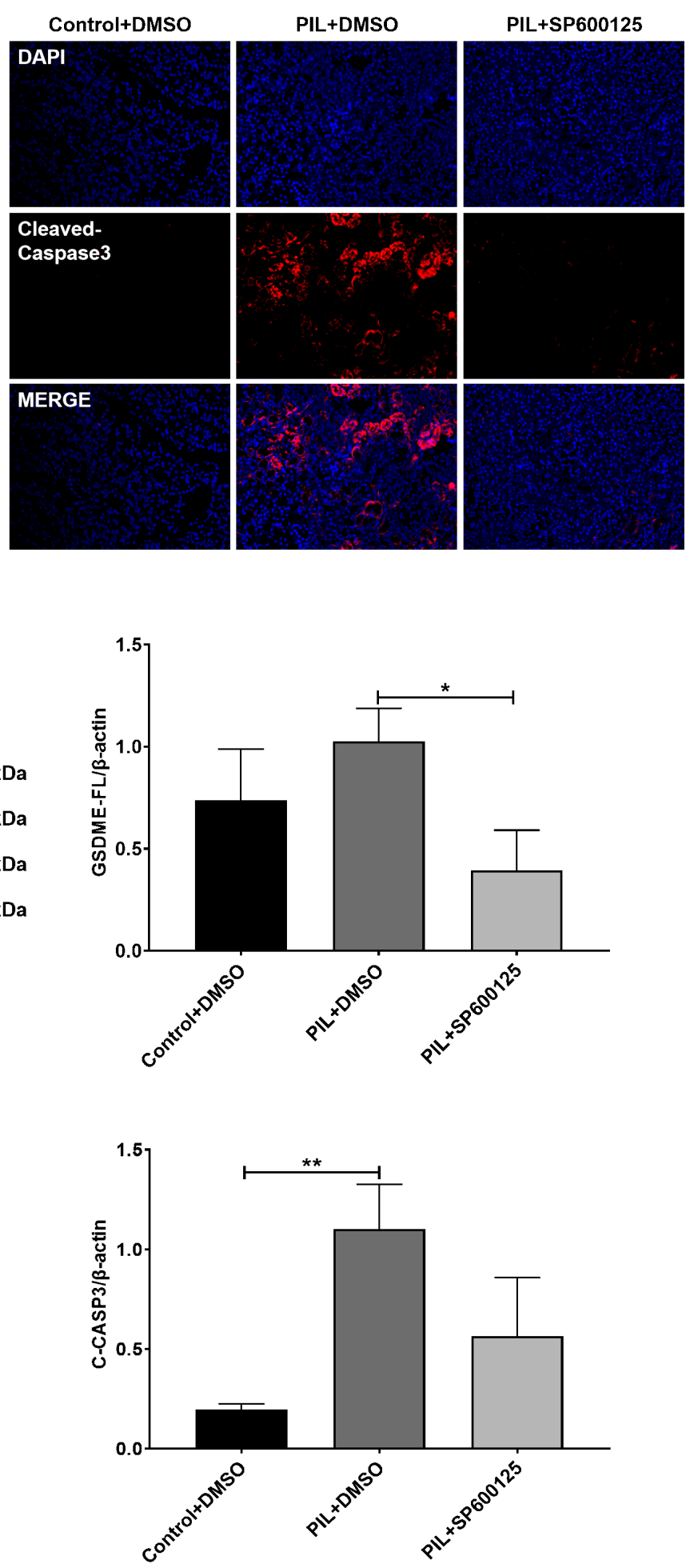

\section{Figure 6}

SP600125 inhibited GSDME mediated secondary pyroptosis in PIL Mice. (A,B)Immunofluorescence staining for GSDME and cleaved caspase-3 in each group mouse kidney were assessed.(C)Western blot and quantitative analysis of the expressions of GSDME and cleaved caspase-3.Data were shown as mean $\pm S D$, ${ }^{*} p<0.05,{ }^{* *} p<0.01$. 